\title{
INTEGRATION BASED ON FUSION FUNCTIONS
}

\author{
L'ubomíra Horanská - Alexandra ŠIPOŠOvá
}

\begin{abstract}
In this paper, we present an approach to data aggregation based on a generalization of the discrete Choquet integral by means of fusion functions. Inspired by Mesiar, R.-Kolesárová, A.-Bustince, G.-Pereira Dimuro, G.-Bedregal, B.: Fusion functions based discrete Choquet-like integrals, European J. Oper. Res. 252 (2016), 601-609, we merge information contained in capacities $m$ of criteria sets and values of score vectors by a fusion function $F$ instead of the product operator. We give the conditions under which fusion functions $F$ yield well-defined functionals $C_{F}^{m}$ and we also discuss properties of these functionals. Some examples for particular capacities $m$ and particular fusion functions $F$ are given.
\end{abstract}

\section{Introduction}

A decision making problem consists in choosing the best alternative according to some criteria. One of the useful tools used for the evaluation of a score vector related to the considered criteria is the Choquet integral, which is able to reflect a certain interaction between criteria. The Choquet integral [2] was generalized in several ways, see, for instance, [4], [5].

Our generalization was inspired by that of Me s i a r et al. in [3], where the authors generalized one of the two usually used discrete forms of the Choquet integral (see below, the formula (10) replacing the product operator by a fusion function satisfying certain conditions. Using the same idea, we generalize the other formula (see the formula (2) ) for the discrete Choquet integral. Note that, in general, the resulting functional differs from that obtained in [3].

We recall the definition of the Choquet integral on a general monotone measure space $(X, \mathfrak{S}, m)$, where $X$ is a non-empty set $X, \mathfrak{S}$ is a $\sigma$-algebra of its subsets and $m: \mathfrak{S} \rightarrow[0, \infty]$ a monotone measure, i.e., a set function satisfying the properties $m(\emptyset)=0$ and $m(A) \leq m(B)$ for all $A, B \in \mathfrak{S}, A \subseteq B$.

(C) 2016 Mathematical Institute, Slovak Academy of Sciences.

2010 Mathematics Subject Classification: 28E10, 62 C99.

Keywords: Choquet integral, capacity, fusion function, aggregation function, decision making.

Supported by the grants APVV-14-0013 and VEGA 1/0420/15. 


\section{L'UBOMÍRA HORANSKÁ - ALEXANDRA ŠIPOŠOVÁ}

Definition 1.1. Let $(X, \mathfrak{S}, m)$ be a monotone measure space. For any $\mathfrak{S}$-measurable function $f: X \rightarrow[0,1]$ the Choquet integral $C h_{m}(f)$ is given by

$$
C h_{m}(f)=\int_{0}^{1} m(\{x \in X \mid f(x) \geq t\}) \mathrm{d} t,
$$

where the integral on the right-hand side is the Riemann integral.

In this paper, we will only deal with finite spaces $X=\{1, \ldots, n\}$ for some $n \in N, n \geq 2, \mathfrak{S}=2^{X}$ and normed monotone measures $m: 2^{X} \rightarrow[0,1]$, i.e., monotone measures with $m(X)=1$, calling them capacities [6]. The set of all capacities $m: 2^{X} \rightarrow[0,1]$ will be denoted by $\mathcal{M}_{n}$. Any $2^{X}$-measurable function $f: X \rightarrow[0,1]$ will be identified with a vector $\mathbf{x}=\left(x_{1}, \ldots, x_{n}\right) \in[0,1]^{n}$, where $x_{i}=f(i), i=1, \ldots, n$.

A discrete form of the Choquet integral is of great importance in decision making theory, regarding a finite set $X=\{1, \ldots, n\}$ as some criteria set, a vector $\mathbf{x} \in[0,1]^{n}$ as a score vector and a capacity $m: 2^{X} \rightarrow[0,1]$ as the weights of particular sets of criteria.

Proposition 1.2. Let $X=\{1, \ldots, n\}$ and let $m: 2^{X} \rightarrow[0,1]$ be a capacity. Then for any $\mathbf{x} \in[0,1]^{n}$ the discrete Choquet integral is given by

$$
\mathcal{C} h_{m}(\mathbf{x})=\sum_{i=1}^{n}\left(x_{(i)}-x_{(i-1)}\right) \cdot m\left(E_{(i)}\right),
$$

where $(\cdot): X \rightarrow X$ is a permutation such that $x_{(1)} \leq \cdots \leq x_{(n)}, E_{(i)}=$ $\{(i), \ldots,(n)\}$ for $i=1, \ldots, n$, and $x_{(0)}=0$, or, equivalently, by

$$
\mathcal{C} h_{m}(\mathbf{x})=\sum_{i=1}^{n} x_{(i)} \cdot\left(m\left(E_{(i)}\right)-m\left(E_{(i+1)}\right)\right),
$$

with $x_{(i)}$ and $E_{(i)}, i=1, \ldots, n$, as above, and $E_{(n+1)}=\emptyset$.

Observe that information contained in a score vector and that one in a capacity are joined by the standard product operator. Replacing the product in formulae (11) and (2) by a function $F:[0,1]^{2} \rightarrow[0,1]$ (a binary fusion function), we obtain the following formulae:

$$
C_{m}^{F}(\mathbf{x})=\sum_{i=1}^{n} F\left(x_{(i)}-x_{(i-1)}, m\left(E_{(i)}\right)\right)
$$

and

$$
C_{F}^{m}(\mathbf{x})=\sum_{i=1}^{n} F\left(x_{(i)}, m\left(E_{(i)}\right)-m\left(E_{(i+1)}\right)\right),
$$

respectively. 
The functionals $C_{F}^{m}$ defined by (3) were deeply studied in [3] including a complete characterization of functionals $C_{m}^{F}$ as aggregation functions.

In this paper, we will analyse the functionals defined by (44). The paper is organized as follows. In the next section, we provide the conditions under which a functional $C_{F}^{m}$ is correctly defined for any capacity $m \in \mathcal{M}_{n}$ and any $\mathbf{x} \in[0,1]^{n}$. The problem has to be solved separately for $n=2$ and $n>2$. In both cases, we also exemplify $C_{F}^{m}$ for suitable fusion functions and for particular capacities. In Section 3, we discuss properties of functionals $C_{F}^{m}$ and show the connection of $C_{F}^{m}$ with the discrete Choquet integral. Finally, some concluding remarks are added.

\section{Functionals $C_{F}^{m}$ : definition and examples}

In this section, we analyse conditions under which the functionals $C_{F}^{m}$ introduced in (4) are well-defined and we bring several examples.

Evidently, for a score vector $\mathbf{x} \in[0,1]^{n}$ with card $\left\{x_{1}, \ldots, x_{n}\right\}=n$ there is a unique permutation (.) : $X \rightarrow X$ such that $x_{(1)} \leq \cdots \leq x_{(n)}$ (in fact, all inequalities are strict). Thus $C_{F}^{m}$ is correctly defined by formula (4). If some ties occur, i.e., if card $\left\{x_{1}, \ldots, x_{n}\right\}<n$, we have to analyse the two following cases.

Case 1: Let $n=2$. Consider $\mathbf{x}=\left(x_{1}, x_{2}\right)=(x, x)$, and a capacity $m_{a, b} \in$ $\mathcal{M}_{2}$ defined by $m_{a, b}(\{1\})=a$ and $m_{a, b}(\{2\})=b$, where $a, b \in[0,1]$. Then $C_{F}^{m_{a, b}}(x, x)$ is well-defined only if formula (44) gives back the same value for both possible permutations $(1,2)$ and $(2,1)$ ordering the vector $\mathbf{x}$ increasingly, i.e., if it holds

$$
F(x, 1-a)+F(x, a)=F(x, 1-b)+F(x, b)
$$

for all $a, b \in[0,1]$.

Consequently, we obtain the following proposition.

Proposition 2.1. Let $n=2$. Then $C_{F}^{m}:[0,1]^{2} \rightarrow[0,2]$ introduced in (4) is well-defined if and only if

$$
F(x, u)+F(x, 1-u)=2 F(x, 1 / 2),
$$

for any $x, u \in[0,1]$.

We can immediately characterize all well-defined functionals $C_{F}^{m_{a, b}}$ :

$$
C_{F}^{m_{a, b}}(x, y)= \begin{cases}F(x, 1-b)+F(y, b) & \text { if } x<y \\ 2 F(x, 1 / 2) & \text { if } x=y \\ F(x, a)+F(y, 1-a) & \text { if } x>y\end{cases}
$$




\section{L'UBOMÍRA HORANSKÁ - ALEXANDRA ŠIPOŠOVÁ}

Example 2.2. Consider $F:[0,1]^{2} \rightarrow[0,1]$ defined by $F(x, y)=\frac{x}{2}\left((2 y-1)^{3}+1\right)$. Then $F$ satisfies the constraints of Proposition 2.1. and thus $C_{F}^{m}$ is correctly defined for any $m_{a, b} \in \mathcal{M}_{2}$. Note that then

$$
C_{F}^{m_{a, b}}(x, y)= \begin{cases}\frac{x+y}{2}+\frac{(y-x)}{2}(2 b-1)^{3} & \text { if } x \leq y, \\ \frac{x+y}{2}+\frac{(x-y)}{2}(2 a-1)^{3} & \text { otherwise. }\end{cases}
$$

If $a=b$, i.e., $m_{a, a}$ is a symmetric capacity, then

$$
C_{F}^{m_{a, a}}(x, y)=\frac{x+y}{2}+\frac{|x-y|}{2}(2 a-1)^{3} \quad \text { for all } \quad x, y \in[0,1] .
$$

Observe that all fusion functions of the form $F(x, u)=f(x) \cdot g(u)$ or $F(x, u)=$ $1-(1-f(x)) \cdot(1-g(u))$, where $f:[0,1] \rightarrow[0,1]$ is an arbitrary function and $g:[0,1] \rightarrow[0,1]$ is such that $g(u)=1-g(1-u)$ for all $u \in[0,1]$, satisfy formula (5) and therefore provide well-defined functionals $C_{F}^{m}$.

The following proposition illustrates that the class of suitable fusion functions contains fusion functions not only of the two types mentioned above. The proof of the proposition is straightforward.

Proposition 2.3. Let $F:[0,1]^{2} \rightarrow[0,1]$ and $G:[0,1]^{2} \rightarrow[0,1]$ be fusion functions satisfying formula (5), and let $\alpha, \beta \in[0,1]$ be such that $\alpha+\beta=1$. Then $\alpha F+\beta G$ defined for all $(x, u) \in[0,1]^{2}$ by

$$
(\alpha F+\beta G)(x, u)=\alpha F(x, u)+\beta G(x, u),
$$

is a fusion function satisfying formula (5).

Moreover, for any capacity $m_{a, b} \in \mathcal{M}_{2}$ it holds that

$$
C_{\alpha F+\beta G}^{m_{a, b}}=\alpha C_{F}^{m_{a, b}}+\beta C_{G}^{m_{a, b}} .
$$

Case 2: Now, consider $n>2$ and a vector $\mathbf{x}=\left(x_{1}, \ldots, x_{n}\right) \in[0,1]^{n}$ such that $\operatorname{card}\left\{x_{1}, \ldots, x_{n}\right\}<n$. Without loss of generality, we can suppose that $\operatorname{card}\left\{x_{1}, \ldots, x_{n}\right\}=n-1$ and $x_{1}=x_{2}=\min \left\{x_{1}, \ldots, x_{n}\right\}=x$. Then, similarly as before, we find out that $C_{F}^{m}(\mathbf{x})$ is well-defined only if

$$
\begin{aligned}
& F(x, 1-m(\{2,3, \ldots, n\}))+F(x, m(\{2,3, \ldots, n\})-m(\{3, \ldots, n\}))= \\
& F(x, 1-m(\{1,3, \ldots, n\}))+F(x, m(\{1,3, \ldots, n\})-m(\{3, \ldots, n\})) .
\end{aligned}
$$

The last equality has to be satisfied for any capacity $m \in \mathcal{M}_{n}$, i.e., for any $\alpha, \beta, \gamma, \delta \in[0,1]$ such that $\alpha+\beta=\gamma+\delta \in[0,1]$ it should hold that

$$
F(x, \alpha)+F(x, \beta)=F(x, \gamma)+F(x, \delta) .
$$

The only solution of this Cauchy's equation (see [1) is of the form

$$
F(x, y)=f(x) \cdot y,
$$

where $f:[0,1] \rightarrow[0,1]$ is an arbitrary function. On the other hand, any function $F$ of the form (17) yields a well-defined functional $C_{F}^{m}:[0,1]^{n} \rightarrow[0, n]$. 


\section{INTEGRATION BASED ON FUSION FUNCTIONS}

Proposition 2.4. Let $n>2$. The functional $C_{F}^{m}:[0,1]^{n} \rightarrow[0, n]$ is well-defined for any $m \in \mathcal{M}_{n}$ if and only if $F(x, u)=f(x) \cdot u$ for all $x, u \in[0,1]$ and some function $f:[0,1] \rightarrow[0,1]$. In that case

$$
C_{F}^{m}(\mathbf{x})=\sum_{i=1}^{n} f\left(x_{(i)}\right) \cdot\left(m\left(E_{(i)}\right)-m\left(E_{(i+1)}\right)\right) .
$$

ExAmple 2.5. Consider $F:[0,1]^{2} \rightarrow[0,1]$ given by $F(x, y)=(1-x) y$ which satisfies Proposition 2.3. Then for each $m \in \mathcal{M}_{n}$ and $\mathrm{x} \in[0,1]^{n}$ it holds:

$$
C_{F}^{m}(\mathbf{x})=1-\mathcal{C} h_{m}(\mathbf{x})=\mathcal{C} h_{m^{d}}(\mathbf{1}-\mathbf{x}),
$$

where $m^{d}$ is a dual capacity to $m$, given by $m^{d}(E)=1-m\left(E^{c}\right)$. Note that $C_{F}^{m}$ is a decreasing operator, $C_{F}^{m}(0, \ldots, 0)=1$ and $C_{F}^{m}(1, \ldots, 1)=0$.

Using (8), for a fixed suitable fusion function $F$ given by (7), we can derive

\begin{tabular}{|c|c|}
\hline$m \in \mathcal{M}_{n}$ & $C_{F}^{m} ; \quad F(x, y)=f(x) \cdot y$ \\
\hline$m^{*}(E)= \begin{cases}1 & \text { if } E \neq \emptyset \\
0 & \text { if } E=\emptyset\end{cases}$ & $C_{F}^{m^{*}}(\mathbf{x})=f\left(x_{(n)}\right)=f\left(\max _{1 \leq i \leq n} x_{i}\right)$ \\
\hline$m_{*}(E)= \begin{cases}1 & \text { if } E=\{1, \cdots, n\} \\
0 & \text { otherwise }\end{cases}$ & $C_{F}^{m_{*}}(\mathbf{x})=f\left(x_{(1)}\right)=f\left(\min _{1 \leq i \leq n} x_{i}\right)$ \\
\hline $\begin{array}{l}m_{H}(E)= \begin{cases}1 & \text { if } H \subseteq E \\
0 & \text { otherwise }\end{cases} \\
\emptyset \neq H \subseteq X\end{array}$ & $\begin{array}{l}C_{F}^{m_{H}}(\mathbf{x})=f\left(x_{i}\right), \text { where } \\
\left\{j \in\{1, \cdots, n\} \mid x_{j} \geq x_{i}\right\} \supseteq H \text { but } \\
\left\{j \in\{1, \cdots, n\} \mid x_{j}>x_{i}\right\} \supseteq H \text { does not hold }\end{array}$ \\
\hline $\bar{m}(E)=\frac{\operatorname{card} E}{n}$ & $C_{F}^{\bar{m}}(\mathbf{x})=\frac{1}{n} \sum_{i=1}^{n} f\left(x_{i}\right)$ \\
\hline
\end{tabular}
$C_{F}^{m}$ for some particular capacities $m \in \mathcal{M}_{n}$, see the following table.

Note that $m^{*}$ and $m_{*}$ are the greatest and the smallest elements of $\mathcal{M}_{n}$, respectively, and that $m_{*}=m_{H}$ for $H=X$. 


\section{L'UBOMÍRA HORANSKÁ - ALEXANDRA ŠIPOŠOVÁ}

\section{Properties of functionals $C_{F}^{m}$}

The Choquet integral is widely used in data aggregation due to its genuine properties. It is desirable for functionals obtained by our generalization to inherit these properties.

Let us recall that a function $A:[0,1]^{n} \rightarrow[0,1]$ is:

- an aggregation function, if $A$ is monotone increasing and

$$
A(\mathbf{0})=A(0, \ldots, 0)=0, \quad A(\mathbf{1})=A(1, \ldots, 1)=1 ;
$$

- a mean, if for each $\mathbf{x} \in[0,1]^{n}$ it satisfies the property

$$
\operatorname{Min}(\mathbf{x}) \leq A(\mathbf{x}) \leq \operatorname{Max}(\mathbf{x}),
$$

where $\operatorname{Min}(\mathbf{x})=\min \left\{x_{1}, \ldots, x_{n}\right\}, \operatorname{Max}(\mathbf{x})=\max \left\{x_{1}, \ldots, x_{n}\right\}$;

- translation invariant, if $A\left(x_{1}+c, \ldots, x_{n}+c\right)=c+A\left(x_{1}, \ldots, x_{n}\right)$ for all $c \in] 0,1]$ and $\left(x_{1}, \ldots, x_{n}\right) \in[0,1]^{n}$ such that $\left(x_{1}+c, \ldots, x_{n}+c\right) \in[0,1]^{n}$;

- idempotent, if $A(x, \ldots, x)=x$ for each $x \in[0,1]$;

- positively homogeneous, if $A(c \mathbf{x})=c A(\mathbf{x})$ for each $\mathbf{x} \in[0,1]^{n}$ and $c>0$ such that $c \mathbf{x} \in[0,1]^{n}$;

- comonotone additive, if $A(\mathbf{x}+\overline{\mathbf{x}})=A(\mathbf{x})+A(\overline{\mathbf{x}})$ for all comonotone vectors $\mathbf{x}, \overline{\mathbf{x}} \in[0,1]^{n}$ such that $\mathbf{x}+\overline{\mathbf{x}} \in[0,1]^{n}$ (vectors $\mathbf{x}=\left(x_{1}, \ldots, x_{n}\right), \overline{\mathbf{x}}=$ $\left(\bar{x}_{1}, \ldots, \bar{x}_{n}\right)$ are comonotone, if $\left(x_{i}-x_{j}\right)\left(\bar{x}_{i}-\bar{x}_{j}\right) \geq 0$ for all $\left.i, j \in\{1, \ldots, n\}\right)$;

Let $n=2$. For binary functionals $C_{F}^{m}$ of the form (6) with $F$ satisfying (5), the following properties can be derived.

Proposition 3.1. Let $n=2$. Let $F:[0,1]^{2} \rightarrow[0,1]$ be a fusion function satisfying formula (5). Then for functional $C_{F}^{m}$ of the form (16) it holds that

(i) $C_{F}^{m}$ is idempotent for each $m \in \mathcal{M}_{2}$, if and only if $F$ satisfies $F\left(x, \frac{1}{2}\right)=\frac{x}{2}$ for all $x \in[0,1]$.

(ii) If $C_{F}^{m}$ is a mean for each $m \in \mathcal{M}_{2}$, then $F\left(x, \frac{1}{2}\right)=\frac{x}{2}$ for all $x \in[0,1]$. Conversely, if $F$ is an increasing function in the first variable, such that $F\left(x, \frac{1}{2}\right)=\frac{x}{2}$ for all $x \in[0,1]$, then $C_{F}^{m}$ is a mean for each $m \in \mathcal{M}_{2}$.

Proof. The idempotency of $C_{F}^{m}$ and the necessity in (ii) follows directly from formula (6).

If $F$ is an increasing function in the first variable such that $F\left(x, \frac{1}{2}\right) \geq \frac{x}{2}$ for all $x \in[0,1]$, using (6), one can easily check that $C_{F}^{m} \geq$ Min for each $m \in \mathcal{M}_{2}$. Analogously, if $F$ is an increasing function in the first variable such that $F\left(x, \frac{1}{2}\right) \leq \frac{x}{2}$ for all $x \in[0,1]$, then $C_{F}^{m} \geq$ Max for each $m \in \mathcal{M}_{2}$. Summarizing, we obtain the sufficient condition in (ii). 


\section{INTEGRATION BASED ON FUSION FUNCTIONS}

Proposition 3.2. Let $n=2$. Let $F:[0,1]^{2} \rightarrow[0,1]$ be a fusion function satisfying formula (5). Then functional $C_{F}^{m}$ is translation invariant for each $m \in \mathcal{M}_{2}$ if and only if $F(x, u)=x g(u)+h(u)$, where $g, h:[0,1] \rightarrow[0,1]$ are functions satisfying the conditions $g(u)+g(1-u)=1, h(u)+h(1-u)=$ $2 F(0,1 / 2), 2 F(0,1 / 2) \leq g(u)+h(u) \leq 1$.

P r o o f. Let $C_{F}^{m}$ be translation invariant. Then, for all $x, y, c, u \in[0,1]$ with $x+c$, $y+c \in[0,1]$, we have

$$
F(x+c, u)+F(y+c, 1-u)=c+F(x, u)+F(y, 1-u) .
$$

Taking $x=y=0$ and $u=1 / 2$, we obtain

$$
F(c, 1 / 2)=c / 2+F(0,1 / 2) .
$$

Thus, for every $x \in[0,1]$, it has to be satisfied

$$
F(x, 1 / 2)=x / 2+F(0,1 / 2),
$$

where $F(0,1 / 2) \in[0,1 / 2]$.

Now, rearranging terms in (9) and using (5), (10), we have

$$
\begin{aligned}
& F(x+c, u)-F(x, u) \\
& =c+F(y, 1-y)-F(y+c, 1-u) \\
& =c+(2 F(y, 1 / 2)-F(y, u))-(2 F(y+c, 1 / 2)-F(y+c, u)) \\
& =F(y+c, u)-F(y, u)+(c+2 y / 2+2 F(0,1 / 2)-2(y+c) / 2-2 F(0,1 / 2)) \\
& =F(y+c, u)-F(y, u) .
\end{aligned}
$$

It means that for the considered $u$ and $c$, it holds that

$$
F(x+c, u)-F(x, u)=F(y+c, u)-F(y, u),
$$

for all admissible $x$ and $y$. It implies that $F(x+c, u)-F(x, u)$ is a function of $c$ and $u$, independent of $x$, i.e.,

$$
F(x+c, u)-F(x, u)=\varphi(c, u) .
$$

Putting consecutively $0, c, 2 c, \ldots$ for $x$, we get

$$
\begin{aligned}
& x=0: F(c, u)-F(0, u)=\varphi(c, u), \\
& x=c: F(2 c, u)=F(c, u)+\varphi(c, u)=2 F(c, u)-F(0, u), \\
& x=2 c: F(3 c, u)=F(2 c, u)+\varphi(c, u)=3 F(c, u)-2 F(0, u),
\end{aligned}
$$

etc. Proceeding by induction, for all $k \in \mathbb{N}, c, u \in[0,1]$ with $k c \in[0,1]$, we obtain

$$
F(k c, u)=k F(c, u)-(k-1) F(0, u),
$$

or, equivalently,

$$
F(k c, u)=k(F(c, u)-F(0, u))+F(0, u) .
$$




\section{L'UBOMÍRA HORANSKÁ - ALEXANDRA ŠIPOŠOVÁ}

For a fixed $u$, we can write $F(k c, u)=H(k c)$ and $H(k c)=k G(c)+t$. Following the technique used in the solution of the Cauchy equation and due to the boundedness of $F$, it can be shown that the last equality holds for every $k \in[0,1]$ (see [1] for details). Hence, we can put $c=1$ and $k=x$, obtaining $H(x)=x G(1)+t$, and consequently from (12) we get

or

$$
F(x, u)=x(F(1, u)-F(0, u))+F(0, u),
$$

$$
F(x, u)=x g(u)+h(u),
$$

where $g(u)=F(1, u)-F(0, u), h(u)=F(0, u)$, and functions $g$, $h$ satisfy the conditions

$$
\begin{aligned}
g(u)+g(1-u) & =1, \\
h(u)+h(1-u) & =2 F(0,1 / 2), \\
2 F(0,1 / 2) & \leq g(u)+h(u) \leq 1 .
\end{aligned}
$$

The necessity is proved.

Now, to prove the sufficiency, consider that $m \in \mathcal{M}_{2}, m(\{1\})=a, m(\{2\})=$ $b, F(x, u)=x g(u)+h(u)$, with $g, h$ satisfying the conditions listed above. Then, if $x<y$, for all admissible $c$ also $x+c<y+c$, and we get

and

$$
\begin{aligned}
& C_{F}^{m}(x+c, y+c) \\
& =F(x+c, 1-b)+F(y+c, b) \\
& =(x+c) g(1-b)+h(1-b)+(y+c) g(b)+h(b) \\
& =c(g(b)+g(1-b))+x g(1-b)+y g(b)+(h(b)+h(1-b)) \\
& =c+x g(1-b)+y g(b)+2 F(0,1 / 2),
\end{aligned}
$$

$$
\begin{aligned}
& C_{F}^{m}(x, y) \\
& =F(x, 1-b)+F(y, b) \\
& =x g(1-b)+h(1-b)+y g(b)+h(b) \\
& =x g(1-b)+y g(b)+2 F(0,1 / 2) .
\end{aligned}
$$

Comparing both expressions, we obtain $C_{F}^{m}(x+c, y+c)=c+C_{F}^{m}(x, y)$. The rest of the proof for $x \geq y$ runs as before.

Proposition 3.3. Let $n=2$. Let $F:[0,1]^{2} \rightarrow[0,1]$ be a fusion function satisfying formula (5). Then functional $C_{F}^{m}$ is comonotone additive for each $m \in \mathcal{M}_{2}$, if and only if $F$ satisfies $F(x, u)=x F(1, u)$ for all $x, u \in[0,1]$.

Proof. Let $C_{F}^{m}$ be comonotone additive for each $m \in \mathcal{M}_{2}$. It means

$$
C_{F}^{m_{a, b}}(\mathbf{x}+\mathbf{y})=C_{F}^{m_{a, b}}(\mathbf{x})+C_{F}^{m_{a, b}}(\mathbf{y})
$$

for all comotonone vectors $\mathbf{x}=\left(x_{1}, x_{2}\right), \mathbf{y}=\left(y_{1}, y_{2}\right)$ with $x_{1}, x_{2}, y_{1}, y_{2} \in[0,1]$. 


\section{INTEGRATION BASED ON FUSION FUNCTIONS}

Due to (6), for every $u \in[0,1]$ it yields

$$
\begin{aligned}
& F\left(x_{1}+y_{1}, u\right)+F\left(x_{2}+y_{2}, 1-u\right)= \\
& \quad F\left(x_{1}, u\right)+F\left(x_{2}, 1-u\right)+F\left(y_{1}, u\right)+F\left(y_{2}, 1-u\right) .
\end{aligned}
$$

For $y_{1}=y_{2}=0$ and $u=1 / 2$ we get

$$
\begin{aligned}
F\left(x_{1}, 1 / 2\right)+F\left(x_{2}, 1 / 2\right) & = \\
F\left(x_{1}, 1 / 2\right) & +F\left(x_{2}, 1 / 2\right)+F(0,1 / 2)+F(0,1 / 2),
\end{aligned}
$$

hence, necessarily $2 F(0,1 / 2)=0$.

Now, from (6) we have

$$
F(0, u)+F(0,1-u)=2 F(0,1 / 2)=0, \quad \text { for all } \quad u \in[0,1],
$$

and the nonnegativity of $F$ implies

$$
F(0, u)=0, \quad \text { for all } \quad u \in[0,1] .
$$

Putting $x_{2}=y_{2}=0, u=1 / 2$, from (13) we get

$$
\begin{aligned}
& F\left(x_{1}+y_{1}, 1 / 2\right)+F(0,1 / 2)= \\
& F\left(x_{1}, 1 / 2\right)+F(0,1 / 2)+F\left(y_{1}, 1 / 2\right)+F(0,1 / 2),
\end{aligned}
$$

which, due to (14), can be written as

$$
F\left(x_{1}+y_{1}, 1 / 2\right)=F\left(x_{1}, 1 / 2\right)+F\left(y_{1}, 1 / 2\right) .
$$

Rearranging terms in (13) and using (15) and (15), we obtain

$$
\begin{aligned}
& F\left(x_{1}+y_{1}, u\right)-F\left(x_{1}, u\right)-F\left(y_{1}, u\right) \\
&= F\left(x_{2}, 1-u\right)+F\left(y_{2}, 1-u\right)-F\left(x_{2}+y_{2}, 1-u\right) \\
&= 2 F\left(x_{2}, 1 / 2\right)-F\left(x_{2}, u\right)+2 F\left(y_{2}, 1 / 2\right)-F\left(y_{2}, u\right) \\
&-\left(2 F\left(x_{2}+y_{2}, 1 / 2\right)-F\left(x_{2}+y_{2}, u\right)\right) \\
&= F\left(x_{2}+y_{2}, u\right)-F\left(x_{2}, u\right)-F\left(y_{2}, u\right) .
\end{aligned}
$$

It follows, that $F(x+y, u)-F(x, u)-F(y, u)$ does not depend on $x, y$, so we can deduce that

$$
F(x+y, u)-F(x, u)-F(y, u)=\varphi(u) .
$$

Moreover, according to (14), for $x=y=0$ we have

Hence

$$
\varphi(u)=F(0, u)-F(0, u)-F(0, u)=0 .
$$

$$
F(x+y, u)=F(x, u)+F(y, u), \quad \text { for all } \quad x, y, u \in[0,1] .
$$

The unique solution of this Cauchy equation is of the form

$$
F(x, u)=x F(1, u), \quad \text { for all } \quad x, u \in[0,1],
$$

and the necessity is proved. 


\section{L'UBOMÍRA HORANSKÁ - ALEXANDRA ŠIPOŠOVÁ}

To prove sufficiency, let $F(x, u)=x F(1, u)$, for all $x, u \in[0,1]$ and let $\mathbf{x}=\left(x_{1}, x_{2}\right), \mathbf{y}=\left(y_{1}, y_{2}\right)$ be comonotone vectors. Then, the vectors $\mathbf{x}, \mathbf{y}, \mathbf{x}+\mathbf{y}$ are ordered increasingly by the same permutation. For $x_{1}<x_{2}$ we have

$$
\begin{aligned}
& C_{F}^{m_{a, b}}(\mathbf{x}+\mathbf{y}) \\
&= F\left(x_{1}+y_{1}, 1-b\right)+F\left(x_{2}+y_{2}, b\right) \\
&=\left(x_{1}+y_{1}\right) F(1,1-b)+\left(x_{2}+y_{2}\right) F(1, b) x_{1} F(1,1-b) \\
&+y_{1} F(1,1-b)+x_{2} F(1, b)+y_{2} F(1, b) \\
&= F\left(x_{1}, 1-b\right)+F\left(x_{2}, b\right)+F\left(y_{1}, 1-b\right)+F\left(y_{2}, b\right) \\
&= C_{F}^{m_{a, b}}(\mathbf{x})+C_{F}^{m_{a, b}}(\mathbf{y})
\end{aligned}
$$

The same formulae can be derived for $x_{1} \geq x_{2}$.

Since comonotone additivity along with boundedness implies positive homogenity, we obtain the following corollary.

Corollary 3.4. Let $n=2$. Let $F:[0,1]^{2} \rightarrow[0,1]$ be a fusion function satisfying formula (5). Then functional $C_{F}^{m}$ is positively homogeneous for each $m \in \mathcal{M}_{2}$, if and only if $F$ satisfies $F(x, u)=x F(1, u)$ for all $x, u \in[0,1]$.

Proposition 3.5. Let $n=2$. Let $F:[0,1]^{2} \rightarrow[0,1]$ be a fusion function satisfying formula (5). Then for functional $C_{F}^{m}$ of the form (6) it holds that

(i) $C_{F}^{m}$ is an aggregation function for each $m \in \mathcal{M}_{2}$ if and only if $F$ is an increasing function in the first variable satisfying $F(0, u)=0$ for all $u \in[0,1]$ and $F\left(1, \frac{1}{2}\right)=\frac{1}{2}$.

(ii) $C_{F}^{m}$ gives back the capacity, i.e., $C_{F}^{m}\left(\chi_{E}\right)=m(E)$ for all $E \subseteq X$ and for each $m \in \mathcal{M}_{2}$, if and only if $F(1, u)=u$ and $F(0, u)=0$ for all $u \in[0,1]$.

P r o o f. Statement (i) follows directly from (6).

To prove (ii), let $F(1, u)=u$ and $F(0, u)=0$ for all $u \in[0,1]$. We can easily check that $C_{F}^{m}\left(\chi_{E}\right)=m(E)$ for all subsets of $X=\{1,2\}$ using (6).

Taking $E=\emptyset$ and supposing that $C_{F}^{m}$ gives back capacity, from ([6) we get

$$
0=C_{F}^{m}\left(\chi_{E}\right)=2 F(0,1 / 2) .
$$

Since $F$ is nonnegative and satisfying

$$
F(0, u)+F(0,1-u)=2 F(0,1 / 2)=0,
$$

we can conclude that $F(0, u)=0$ for all $u \in[0,1]$.

Take $E=\{1\}$. Considering such capacity $m \in \mathcal{M}_{2}$, that $m(\{1\})=u$ for fixed $u \in[0,1]$, from (6) we obtain

$$
u=C_{F}^{m}\left(\chi_{E}\right)=F(1, u)+F(0,1-u)=F(1, u),
$$

and the necessity is proved. 


\section{INTEGRATION BASED ON FUSION FUNCTIONS}

Now, we focus on the symmetry of binary functionals $C_{F}^{m}$. Obviously, for a symmetric capacity $m_{a, a}$ we obtain a symmetric functional $C_{F}^{m_{a, a}}$. Moreover, it holds that

$$
C_{F}^{m_{a, a}}(x, y)=F(\min \{\mathrm{x}, \mathrm{y}\}, 1-a)+F(\max \{\mathrm{x}, \mathrm{y}\}, a) .
$$

This functional can be regarded as a generalization of the OWA operator defined by

$$
O W A(x, y)=(1-a) \min \{\mathrm{x}, \mathrm{y}\}+\operatorname{amax}\{\mathrm{x}, \mathrm{y}\} .
$$

Note that for obtaining a symmetric functional $C_{F}^{m}$, the considered capacity need not be necessarily symmetric, see the following example.

EXAmple 3.6. Let $F(x, u)=f(x)+g(u)$, where $f:[0,1] \rightarrow\left[0, \frac{1}{2}\right]$ is an arbitrary function and $g:[0,1] \rightarrow\left[0, \frac{1}{2}\right]$ is such that $g(u)+g(1-u)=c$ for some $c \in\left[0, \frac{1}{2}\right]$. Then $F$ satisfies formula (5),$C_{F}^{m_{a, b}}$ is correctly defined for any $m_{a, b} \in \mathcal{M}_{2}$, and it can be expressed as

$$
C_{F}^{m_{a, b}}(x, y)=f(x)+f(y)+2 c,
$$

which is a symmetric functional.

Now, let $n \geq 2$. Since a fusion function satisfying (7) fulfils also (15), the class of all suitable fusion functions yielding well-defined binary functional $C_{F}^{m}$ contains all suitable fusion functions yielding well-defined functional in higher dimensions. Thus the following results for the functionals $C_{F}^{m}$ of the form (8) with $F$ satisfying (7) for $n=2$ are special cases of those in the previous propositions.

Proposition 3.7. Let $F:[0,1]^{2} \rightarrow[0,1], F(x, u)=f(x) u$, where $f:[0,1] \rightarrow[0,1]$ is an arbitrary function. Then for any fixed $n \geq 2$ it holds that

(i) $C_{F}^{m}$ is a mean for each $m \in \mathcal{M}_{n}$ if and only if $f(x)=x$ for all $x \in[0,1]$.

(ii) $C_{F}^{m}$ is idempotent for each $m \in \mathcal{M}_{n}$, if and only if $f(x)=x$ for all $x \in[0,1]$.

(iii) $C_{F}^{m}$ is translation invariant for each $m \in \mathcal{M}_{n}$, if and only if $f(x)=x$ for all $x \in[0,1]$.

Proof.

(i) Using (8), it can be proved that $C_{F}^{m} \geq$ Min for each $m \in \mathcal{M}_{n}$, if and only if $f(x) \geq x$. Similarly, $C_{F}^{m} \leq$ Max for each $m \in \mathcal{M}_{n}$, if and only if $f(x) \leq x$. Summarizing, we obtain the statement.

(ii) The statement follows directly from (8).

(iii) Let $f(x)=x$ for all $x \in[0,1]$. The same permutation orders increasingly vectors $\mathbf{x}=\left(x_{1}, \ldots, x_{n}\right)$ and $\left(x_{1}+c, \ldots, x_{n}+c\right)$ for every $\left.\left.c \in\right] 0,1\right]$. 


\section{L'UBOMÍRA HORANSKÁ - ALEXANDRA ŠIPOŠOVÁ}

Thus

$$
\begin{aligned}
& C_{F}^{m}\left(x_{1}+c, \ldots, x_{n}+c\right) \\
& =\sum_{i=1}^{n}\left(x_{(i)}+c\right)\left(m\left(E_{(i)}\right)-m\left(E_{(i+1)}\right)\right) \\
& =\sum_{i=1}^{n} x_{(i)}\left(m\left(E_{(i)}\right)-m\left(E_{(i+1)}\right)\right)+c \sum_{i=1}^{n}\left(m\left(E_{(i)}\right)-m\left(E_{(i+1)}\right)\right) \\
& =C_{F}^{m}\left(x_{1}, \ldots, x_{n}\right)+c .
\end{aligned}
$$

Conversely, let $C_{F}^{m}$ be a translation invariant functional. Then for every $c \in] 0,1]$ and $x_{1}, \ldots, x_{n} \in[0,1]$ it holds

$$
C_{F}^{m}\left(x_{1}+c, \ldots, x_{n}+c\right)=C_{F}^{m}\left(x_{1}, \ldots, x_{n}\right)+c .
$$

Considering $x_{1}=\cdots=x_{n}=0$ and using (8) we obtain

$$
\begin{aligned}
C_{F}^{m}(c, \ldots, c) & =\sum_{i=1}^{n} f(c)\left(m\left(E_{(i)}\right)-m\left(E_{(i+1)}\right)\right) \\
& =f(c) \sum_{i=1}^{n}\left(m\left(E_{(i)}\right)-m\left(E_{(i+1)}\right)\right)=f(c),
\end{aligned}
$$

and

$$
\begin{aligned}
c+C_{F}^{m}(0, \ldots, 0) & =c+\sum_{i=1}^{n} f(0)\left(m\left(E_{(i)}\right)-m\left(E_{(i+1)}\right)\right) \\
& =c+f(0) \sum_{i=1}^{n}\left(m\left(E_{(i)}\right)-m\left(E_{(i+1)}\right)\right)=c+f(0) .
\end{aligned}
$$

Due to the translation invariance of $C_{F}^{m}$ it means

$$
f(c)=c+f(0), \quad \text { for all } \quad c \in] 0,1] .
$$

Since $f(x) \leq 1$ for every $x \in[0,1]$, putting $c=1$ in (16) we can conclude that $f(0)=0$ and consequently $f(x)=x$ for all $x \in[0,1]$.

Note that for the standard product $F(x, u)=x u$ the functional $C_{F}^{m}$ coincides with $\mathcal{C} h_{m}$, therefore the properties of being a mean, idempotent and translation invariant hold only for the Choquet integral itself.

Proposition 3.8. Let $F:[0,1]^{2} \rightarrow[0,1], F(x, u)=f(x) u$, where $f:[0,1] \rightarrow[0,1]$ is an arbitrary function. Then for any fixed $n \geq 2, C_{F}^{m}$ is comonotone additive for each $m \in \mathcal{M}_{n}$, if and only if $f$ satisfies $f(x)=x f(1)$ for all $x \in[0,1]$. 
Proof. Let $f(x)=x f(1)$ for all $x \in[0,1]$ and let $\mathbf{x}=\left(x_{1}, \ldots, x_{n}\right)$ and $\mathbf{x}^{\prime}=\left(x_{1}^{\prime}, \ldots, x_{n}^{\prime}\right)$ be comonotone vectors. Then, the same permutation orders vectors $\mathbf{x}, \mathbf{x}^{\prime}, \mathbf{x}+\mathbf{x}^{\prime}$ increasingly. Hence,

$$
\begin{aligned}
& C_{F}^{m}\left(\mathbf{x}+\mathbf{x}^{\prime}\right) \\
& =\sum_{i=1}^{n} f\left(x_{(i)}+x_{(i)}^{\prime}\right)\left(m\left(E_{(i)}\right)-m\left(E_{(i+1)}\right)\right) \\
& =f(1) \sum_{i=1}^{n}\left(x_{(i)}+x_{(i)}^{\prime}\right)\left(m\left(E_{(i)}\right)-m\left(E_{(i+1)}\right)\right) \\
& =f(1) \sum_{i=1}^{n}\left(x_{(i)}\right)\left(m\left(E_{(i)}\right)-m\left(E_{(i+1)}\right)\right)+f(1) \sum_{i=1}^{n}\left(x_{(i)}^{\prime}\right)\left(m\left(E_{(i)}\right)-m\left(E_{(i+1)}\right)\right) \\
& =\sum_{i=1}^{n} f\left(x_{(i)}\right)\left(m\left(E_{(i)}\right)-m\left(E_{(i+1)}\right)\right)+\sum_{i=1}^{n} f\left(x_{(i)}^{\prime}\right)\left(m\left(E_{(i)}\right)-m\left(E_{(i+1)}\right)\right) \\
& =C_{F}^{m}(\mathbf{x})+C_{F}^{m}\left(\mathbf{x}^{\prime}\right) .
\end{aligned}
$$

Conversely, considering vectors $\mathbf{x}=(x, \ldots, x), \mathbf{x}^{\prime}=\left(x^{\prime}, \ldots, x^{\prime}\right)$, for a comonotone additive functional $C_{F}^{m}$ we have

$$
\begin{aligned}
& \sum_{i=1}^{n} f\left(x+x^{\prime}\right)\left(m\left(E_{(i)}\right)-m\left(E_{(i+1)}\right)\right) \\
& =C_{F}^{m}\left(\mathbf{x}+\mathbf{x}^{\prime}\right) \\
& =C_{F}^{m}(\mathbf{x})+C_{F}^{m}\left(\mathbf{x}^{\prime}\right) \\
& =\sum_{i=1}^{n} f(x)\left(m\left(E_{(i)}\right)-m\left(E_{(i+1)}\right)\right)+\sum_{i=1}^{n} f\left(x^{\prime}\right)\left(m\left(E_{(i)}\right)-m\left(E_{(i+1)}\right)\right) .
\end{aligned}
$$

Therefore,

$$
\begin{aligned}
0 & =\sum_{i=1}^{n}\left(f\left(x+x^{\prime}\right)-f(x)+f\left(x^{\prime}\right)\right)\left(m\left(E_{(i)}\right)-m\left(E_{(i+1)}\right)\right) \\
& =\left(f\left(x+x^{\prime}\right)-f(x)+f\left(x^{\prime}\right)\right) \sum_{i=1}^{n}\left(m\left(E_{(i)}\right)-m\left(E_{(i+1)}\right)\right) \\
& =f\left(x+x^{\prime}\right)-f(x)+f\left(x^{\prime}\right) .
\end{aligned}
$$

Thus, we obtain the Cauchy equation

$$
f\left(x+x^{\prime}\right)=f(x)+f\left(x^{\prime}\right), \quad \text { for all } \quad x, x^{\prime} \in[0,1]
$$

with the unique solution in form $f(x)=x f(1)$. 


\section{L'UBOMÍRA HORANSKÁ - ALEXANDRA ŠIPOŠOVÁ}

Corollary 3.9. Let $F:[0,1]^{2} \rightarrow[0,1], F(x, u)=f(x) u$, where $f:[0,1] \rightarrow[0,1]$ is an arbitrary function. Then for any fixed $n \geq 2, C_{F}^{m}$ is positively homogeneous for each $m \in \mathcal{M}_{n}$, if and only if $f$ satisfies $f(x)=x f(1)$ for all $x \in[0,1]$.

Proposition 3.10. Let $F:[0,1]^{2} \rightarrow[0,1], F(x, u)=f(x) u$, where $f:[0,1] \rightarrow[0,1]$ is an arbitrary function. Then for any fixed $n \geq 2$ it holds that

(i) $C_{F}^{m}$ is an aggregation function for each $m \in \mathcal{M}_{n}$ if and only if $f$ is an increasing function satisfying $f(0)=0$ and $f(1)=1$.

(ii) $C_{F}^{m}$ gives back the capacity for each $m \in \mathcal{M}_{n}$, if and only if $f(0)=0$ and $f(1)=1$.

Proof.

(i) Due to (8),$f(0)=0$ and $f(1)=1$ is equivalent to $C_{F}^{m}(\mathbf{0})=0$ and $C_{F}^{m}(\mathbf{1})=1$, respectively. Moreover, the increasingness of a function $f$ is equivalent to the increasingness of $C_{F}^{m}$ in each variable.

(ii) For a fusion function $F(x, u)=f(x) u$, the functional $C_{F}^{m}$ is in form (8)). Let $E \subseteq X$ be such that $\operatorname{card}(E)=k$. Then $E_{(n-k+1)}=E$, where $(\cdot)$ is a permutation ordering the vector $\chi_{E}$ increasingly. Then

$$
\begin{aligned}
& C_{F}^{m}\left(\chi_{E}\right) \\
& =\sum_{i=1}^{n-k} f(0)\left(m\left(E_{(i)}\right)-m\left(E_{(i+1)}\right)\right)+\sum_{i=n-k+1}^{n} f(1)\left(m\left(E_{(i)}\right)-m\left(E_{(i+1)}\right)\right) \\
& =f(0)\left(m\left(E_{(1)}\right)-m\left(E_{(n-k)}\right)\right)+f(1) m\left(E_{(n-k+1)}\right) \\
& =f(0)\left(1-m\left(E_{(n-k)}\right)\right)+f(1) m(E) .
\end{aligned}
$$

Now, the sufficiency is clear.

For every $y, z \in[0,1]$ there exists such capacity $m \in \mathcal{M}_{n}$, that $m\left(E_{(n-k)}\right)=y$ and $m(E)=z$. Therefore, supposing $C_{F}^{m}\left(\chi_{E}\right)=m(E)$, from the previous equality it follows

$$
f(0)(1-y)+f(1) z=z, \quad \text { for all } \quad y, z \in[0,1]
$$

or equivalently,

$$
f(0)(1-y)=z(1-f(1)), \quad \text { for all } y, z \in[0,1] .
$$

It implies $f(0)=0$ and $f(1)=1$, thus we have finished the proof.

Since an increasing function preserves ordering of an input vector and a decreasing one inverts it, we obtain the following propositions that show the connection between $C_{F}^{m}$ and the discrete Choquet integral. 
Proposition 3.11. Let $F:[0,1]^{2} \rightarrow[0,1], F(x, y)=f(x)$ y, where $f:[0,1] \rightarrow$ $[0,1]$ is an increasing function. Then, for each $m \in \mathcal{M}_{n}$ and $\mathbf{x} \in[0,1]^{n}$,

$$
C_{F}^{m}(\mathbf{x})=\mathcal{C} h_{m}(f(\mathbf{x}))
$$

where $f(\mathbf{x})=\left(f\left(x_{1}\right), \ldots, f\left(x_{n}\right)\right)$.

Proposition 3.12. Let $F:[0,1]^{2} \rightarrow[0,1], F(x, y)=f(x) y$, where $f:[0,1] \rightarrow[0,1]$ is a decreasing function. Then, for each $m \in \mathcal{M}_{n}$ and $\mathbf{x} \in[0,1]^{n}$,

$$
C_{F}^{m}(\mathbf{x})=1-\mathcal{C} h_{m}(1-f(\mathbf{x}))=\mathcal{C} h_{m^{d}}(f(\mathbf{x}))
$$

where

$$
m^{d} \in \mathcal{M}_{n}
$$

is a capacity dual to $m$.

Note that the last property was already illustrated for a special function $F$ in Example 2.5.

\section{Concluding remarks}

We have generalized the formula (2) for the discrete Choquet integral replacing the standard product operator by a function $F:[0,1]^{2} \rightarrow[0,1]$. Several particular operators $C_{F}^{m}$ were discussed, based either on a fixed capacity $m \in \mathcal{M}_{n}$ or on a fixed function $F$. We have found the conditions under which a fusion function $F$ yields a well-defined functional $C_{F}^{m}$, with desirable properties of fuzzy integrals. It is worth mentioning that the class of functionals $C_{F}^{m}$ is much richer in the binary case than in the higher dimensions. Moreover, for $n>2$ the genuine properties of being translation invariant, idempotent or a mean are fulfilled just for the Choquet integral itself. We expect applications of our results in all domains where the generalizations of the discrete Choquet integral are considered, for example in medicine.

\section{REFERENCES}

[1] ACZÉL, J.: Lectures on Functional Equations and their Applications. Academic Press, New York, 1966.

[2] CHOQUET, G.: Theory of capacities, Ann. Inst. Fourier 5 (1953-54), 131-295.

[3] Mesiar, R.-KOlesárová, A.-Bustince, G.-PereirA Dimuro, G.-BEDREGAL, B.: Fusion functions based discrete Choquet-like integrals, European J. Oper. Res. 252 (2016), 601-609.

[4] MESIAR, R.: Choquet-like integrals, J. Math. Anal. Appl. 194 (1995), 477-488. 


\section{L'UBOMÍRA HORANSKÁ - ALEXANDRA ŠIPOŠOVÁ}

[5] KLEMENT, E. P.-MESIAR, R.-PAP, E.: A universal integral as common frame for Choquet and Sugeno integral, IEEE Trans. Fuzzy Systems 18 (2010), 178-187.

[6] GRABISCH, M.-MARICHAL, J.-L.-MESIAR, R.-PAP, E.: Aggregation Functions. Cambridge University Press, Cambridge, 2009.

Received July 15, 2016

\author{
Lubomíra Horanská \\ Institute of Information Engineering, \\ Automation and Mathematics \\ Faculty of Chemical and \\ Food Technology \\ Slovak University of Technology \\ in Bratislava, \\ Radlinského 9 \\ SK-812-37 Bratislava \\ SLOVAKIA
}

E-mail: lubomira.horanska@stuba.sk

Alexandra Šipošová

Department of Mathematics and

Descriptive Geometry

Faculty of Civil Engineering

Slovak University of Technology

in Bratislava

Radlinského 11

SK-810-05 Bratislava

SLOVAKIA

E-mail: alexandra.siposova@stuba.sk 